\title{
A Arte como Estratégia de Coping em Tempos de Pandemia
}

\author{
Art as a Coping Strategy During the Pandemic
}

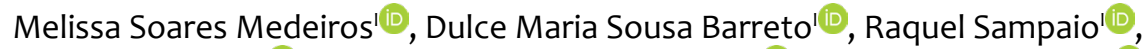 \\ Bruno Cavalcante Fales de Brito Alves ${ }^{10}$, Deborah Costa Moreira Albino' ${ }^{\mathbb{0}}$, lana Lima Fernandes
}

\begin{abstract}
RESUMO
Introdução: O momento da pandemia por Covid-19 tem um impacto direto na formação dos estudantes de Medicina, tanto pela modificação repentina na metodologia presencial para remota quanto pelo estresse e pela ansiedade gerados. A saúde mental dos estudantes necessita de estratégias de coping para que eles possam lidar com situações de extrema ansiedade.

Relato de experiência: O grupo Estudo de Literatura e Arte na Medicina (Elam) criou, durante o período da pandemia, momentos de discussão de literatura por meio de produção textual e discussões em mídia social, e principalmente por meio de atividades artísticas com pintura em aquarela e artes plásticas, para lidar com os conflitos apresentados pelos alunos na quarentena. Foram 24 telas de aquarela, vídeos, telas de colagem em artes plásticas e textos de crônicas e poesias. Os temas abordados nos textos foram principalmente: amor ( $\mathrm{N}=2)$, envelhecer ( $\mathrm{N}$ $=2)$, pecado e religiosidade $(\mathrm{N}=4)$, ciclo da vida $(\mathrm{N}=6)$, câncer, caos, sabedoria, mortalidade $(\mathrm{N}=3)$, tempo, olhar e ver $(\mathrm{N}=2)$ e regionalismo $(\mathrm{N}=2)$. Discussão: $O$ enfrentamento da Covid-19 impõe o isolamento social, o distanciamento físico e a restrição à mobilidade das pessoas como medidas fundamentais para evitar a rápida disseminação do vírus. Quando o jovem não dispõe de mecanismos para lidar com a situação estressante propiciada pela situação, pode haver o desencadeamento de uma série de psicopatologias, como depressão, ansiedade e distúrbios do humor. É uma tendência mais ampla na educação médica: cada vez mais, as escolas de Medicina estão investindo em currículo e programação em torno das artes, e, no momento atual, essa estratégia de coping se torna importante no enfrentamento da pandemia pelo estudante de Medicina. Conclusão: Atravessar esse momento de pandemia pela Covid-19 exigiu uma estratégia de coping para ajudar os alunos a vivenciar e extravasar seus medos, sendo a arte a melhor forma de expressão, o que foi atingido pelo grupo Elam.
\end{abstract}

Palavras-chave: Arte; Literatura; Doença Mental; Pandemia.

\begin{abstract}
Introduction: The Covid-19 pandemic has had a direct impact on medical student training, through the sudden change from face-to-face to remote learning and the stress and anxiety caused. Students need coping strategies for the sake of their mental health and to deal with situations of extreme anxiety. Experience Report: during the pandemic the ELAM (Study of Literature and Art in Medicine) group has sought to help students in lockdown deal with their mental struggles by promoting discussions on literature through texts it has produced, debates on social media and, primarily, through art activities such as watercolor painting and visual arts. Twenty-four watercolor paintings, videos, collages, chronicles and poems were produced. The main themes addressed were: love ( $\mathrm{N}=2)$, ageing $(\mathrm{N}=2)$, sin and religion ( $\mathrm{N}=4)$, life cycle $(\mathrm{N}=6)$, cancer, chaos, wisdom, mortality ( $\mathrm{N}=3)$, time, looking and seeing $(\mathrm{N}=2)$ and regionalism $(\mathrm{N}=2)$.

Discussion: Essential measures to tackle Covid-19 include social isolation, physical distancing and restrictions on people's movements to prevent the rapid spread of the virus. When youngsters lack the mechanisms to cope with the resulting stressful situation, a series of mental disorders can be triggered, such as depression, anxiety and mood swings. Reflecting a broader trend in medical training, medical schools are increasingly investing in curricular content involving arts, and at this particular moment in time this represents an important coping strategy for students trying to deal with the pandemic. Conclusion: Getting through this difficult period of the Covid-19 pandemic has demanded a coping strategy to help students experience and vent their fears, where art has been the best form of expression, and the ELAM group has achieved such a strategy.
\end{abstract}

Keywords: Art; Literature; Mental Illness; Pandemic.

'Centro Universitário Christus, Fortaleza, Ceará, Brasil.

Correspondência:

Melissa Soares Medeiros.

Rua Professor Jacinto Botelho 650, ap. 202, Guararapes, Fortaleza, CE, Brasil. CEP: 60810-050.

E-mail: melmedeiros@hotmail.com

Recebido em 11/08/20; Aceito em 19/08/20. 


\section{INTRODUÇÃO}

Imensuráveis serão os danos deixados pela pandemia causada pela coronaviris disease 2019 (Covid-19) no mundo. Um capítulo da história da humanidade em andamento, em que novamente a população mais vulnerável se torna a maior vítima. As pessoas com maior risco de infecção por coronavírus geralmente vivem na interseção de múltiplas formas de vulnerabilidade e instabilidade estrutural, em situações precárias que se equilibram entre doenças crônicas, desafios de saúde mental, racismo, pobreza, uso de substâncias e traumas. Entre 2000 e 2014, 324 estudantes (220 homens e 104 mulheres) morreram durante a residência nos Estados Unidos, sendo o suicídio a principal causa dos homens'. Há relatos de que a taxa de suicídio entre médicos do sexo masculino é $40 \%$ maior do que entre os homens na população em geral, enquanto a taxa entre as médicas é $130 \%$ maior do que entre as mulheres na população em geral ${ }^{2}$. Curiosamente, o aumento do risco de suicídio entre os médicos pode começar durante a graduação³. Uma abundância de pesquisas revela que estudantes de Medicina têm uma qualidade de vida mental substancialmente inferior à dos indivíduos com idades semelhantes na população em geral ${ }^{4}$. Um estudo de revisão sistemática de 57 trabalhos que avaliaram cerca de 18.015 estudantes de Medicina no Brasil encontrou prevalência de depressão entre eles de 30,6\%. Outros transtornos identificados foram estresse psicológico, ansiedade, distúrbios do padrão de sono, esgotamento, distúrbios alimentares e uso potencialmente perigoso de álcool. O problema de saúde mental mais prevalente que se enquadrou na metanálise foi traço de ansiedade $(89,6 \%)$, indicando que a maioria dos estudantes de Medicina tem uma tendência consideravelmente permanente de sentir ansiedade, estresse e preocupações 5 .

A saúde mental dos profissionais de saúde preocupa há algumas décadas, considerando o caráter estressante do trabalho deles. A preocupação com a saúde mental entre os médicos, principalmente no momento de pandemia, é crescente, pois, além de recair sobre eles grande parte da expectativa de cura dos pacientes, enfrentam os dilemas éticos de possíveis escolhas a serem feitas em situações de urgência. Trabalhar a estabilidade emocional e mental dos estudantes dentro do ambiente universitário se torna uma necessidade premente e inquestionável. Nesse contexto de angústia, dor e desejo de remissão, o aluno vive envolvido em extrema carga de trabalho de estudos e atividades de estágio ${ }^{6}$. Contudo, a Covid-19 os encarcerou em casa e, nesse processo, trouxe a pressão psicológica com a modalidade de ensino remoto, métodos de avaliação on-line e enfrentamento da sobrecarga social e econômica familiar, que não amenizaram o contexto psicológico com que costumeiramente se deparam presencialmente na instituição.

Estudo realizado na Universidade Estadual do Rio Grande do Norte (Uern) concluiu que a maioria dos alunos se sente sobrecarregada com atividades curriculares $e$ complementares, com pouco tempo para o lazer. Apesar disso, muitos se engajam em atividades extracurriculares e buscam aprimorar seu currículo profissional e adquirir novas competências técnicas. Essas atividades podem causar impactos negativos ao reduzirem a qualidade de vida?.

Nesse sentido, surgiu o grupo Estudo de Literatura e Arte na Medicina (Elam), composto de professores que sempre se reuniam antes das aulas de tutoria da Faculdade de Medicina do Centro Universitário Christus (Unichristus). Durante a despedida do semestre em 2019, o grupo resolveu abordar com os alunos questões referentes à literatura e à arte. O nome Elam surgiu da modificação da palavra "elã” que significa "inspiração, criatividade ou suspiro criador" ou ainda "princípio que explica a evolução da vida nas suas mais variadas formas". A proposta era levar literatura e arte para a medicina, de modo a estimular os alunos a enriquecer a leitura, amadurecer a empatia e a ética, desenvolver melhor a coleta de história clínica do paciente, permitir o aprimoramento do raciocínio e conectar a vida com a arte. Pelo processo artístico e literário, os alunos aprenderiam a se comunicar melhor com os pacientes, por meio do desenvolvimento de informações sobre assuntos relacionados à cultura e à diversidade do pensar e viver.

\section{RELATO DE EXPERIÊNCIA}

A seleção inicial para o Elam foi de 40 alunos, e, ao final de 2019, as reuniões com professores ligados à literatura e à arte aconteciam na segunda terça-feira de cada mês. Em janeiro de 2020, a pandemia provocada pelo novo coronavírus se anunciou, e, embora já soubéssemos de sua existência, mantivemos a esperança de que ela não nos alcançaria, assim como aconteceu com a síndrome respiratória aguda severa (severe acute respiratory syndrome - Sars) e a síndrome respiratória do Oriente Médio (Middle East respiratory syndrome - Mers). Estávamos organizando uma atividade para produção em aquarela com os alunos, vinculada a um texto sobre suas produções ao final de fevereiro de 2020, quando tivemos nossa última reunião. Nesse momento, foi solicitado aos alunos que expressassem seus sentimentos e suas emoções da melhor forma por meio da pintura, e posteriormente eles produziriam seus textos.

No total, produziram-se 24 telas em aquarela. Antes do início da atividade, um dos coordenadores preparou um tutorial sobre como utilizar a tinta de aquarela e estratégias 
simples para conseguir tons e efeitos de textura. Durante duas horas, os alunos produziram as telas com as mais variadas temáticas. Após a realização da pintura, elaboraram-se os textos no transcorrer da pandemia. Os temas abordados nos textos foram principalmente: amor $(\mathrm{N}=2)$, envelhecer $(\mathrm{N}=2)$, pecado e religiosidade $(N=4)$, ciclo da vida $(N=6)$, câncer, caos, sabedoria, mortalidade $(\mathrm{N}=3)$, tempo, olhar e ver $(\mathrm{N}=2)$ e regionalismo $(N=2)$ (Figura 1).

A seguir, apresentamos uma pequena mostra dos textos sobre a pandemia, nos quais se evidenciam o sentimento do grupo e a necessidade de cura neste momento de angústia e sofrimento.

\section{Texto1}

(Pan)demia

PAN de sentimentos, infelizmente, em sua maioria, negativos.

Ansiedade, medo, tristeza, insegurança, "realmente estamos vivendo isso?".

Mas também com oscilações de fé, perdão, prosperidade, compaixão.

Responsabilidade.

Comigo. Com o próximo. Com o Brasil. Com o mundo. Com a saúde.

Incertezas continuam.

Virá uma "segunda onda"?

Ansiedade, insegurança, prosperidade.

Pan demia.

Texto 2

Em meio ao caos, a paz e a calmaria, um tanto árcades, se tornam os objetivos mais profundos da raça humana. Em meio ao caos, a liberdade é o que se busca incansavelmente, a cada segundo.

Em meio ao caos, pode-se finalmente dar valor ao que realmente importa.

A vida é um breve sopro.

Um suspiro

Texto 3

Me sinto doente.

0

T e m po

$T$

O

D

O

Me sinto sem forças.

A vitalidade se esvaiu de mim, como os grãos de areia da praia que eu não vou se esvaem das minhas mãos.

Como se foi a vida do leito 274 sem que nada pudesse ser feito. Restou apenas seu prontuário.

Assim como de mim restaram apenas documentos E um diploma

Texto 4

No anoitecer

A lua ilumina o caminho

Da travessia.

Entre cordilheiras há um rio.
Figura 1. "Olha as cores! Vê o contraste! Não vês de cima? Que chegaste? Alma plena, sangrenta. Em cena."

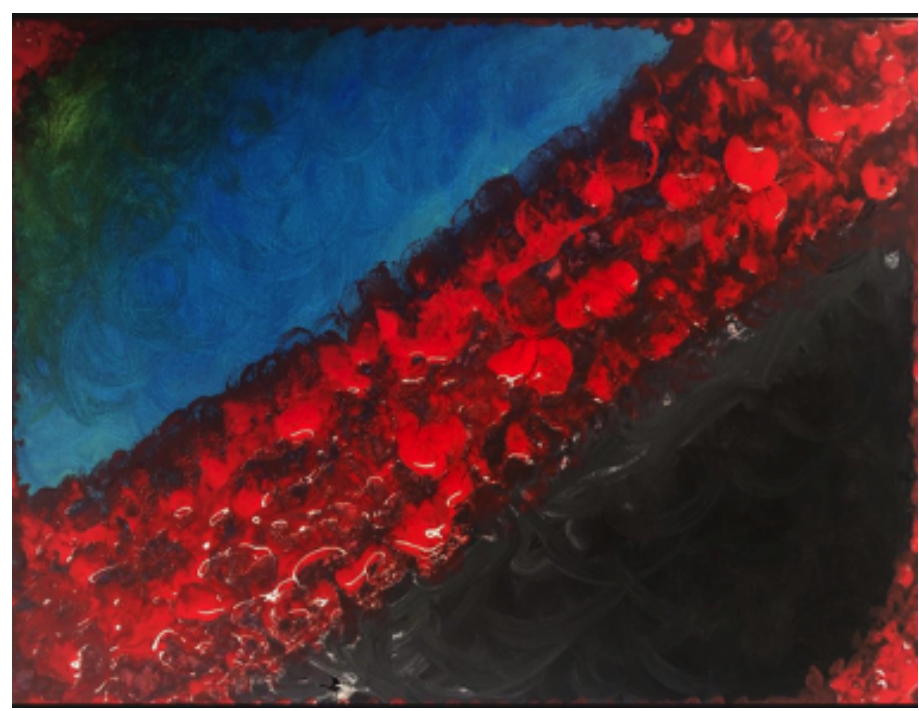

Fonte: Elaborada por participante do grupo Elam
Frio, Medo, Receio
Sem saber o q esperar
Quando enfim atravessar.
Em meio aos atravancos
Haverá um novo rumo
Uma nova vida,
Um novo ser
Q virá um dia padecer
E se lembrará q venceu
os óbices da sua existência.

Os 24 textos sobre as aquarelas foram reunidos e transformados em nuvem de palavras, refletindo as que mais prevaleceram (Figura 2). Por meio dos textos, o aluno pôde trabalhar uma estratégia de coping diferente, menos estressante, que lhe permitisse extravasar os sentimentos e as ansiedades para um grupo. O conceito de coping é derivado de duas abordagens: a primeira, vinculada à experimentação animal, refere-se ao comportamento de fuga e esquiva; e a segunda, relacionada à psicologia do ego, foca os mecanismos de defesa propostos pela psicanálise ${ }^{8}$. Em uma visão mais integradora, o coping é explicado como um processo transacional entre a pessoa e o ambiente, em que as estratégias de enfrentamento têm as funções de alterar a relação entre a pessoa e o ambiente e adequar a resposta emocional ao problema9.

Com o ambiente virtual, as reuniões ficaram mais próximas, apesar do período reduzido de tempo (entre 40 e 60 minutos), e ocorriam a cada 15 dias. Nesse período, além da discussão sobre literatura, criou-se uma página no Instagram para divulgar as imagens e os textos do grupo (@ elam.unichristus), e realizaram-se lives com os professores relacionados às humanidades médicas. Estimulou-se ainda 
uma maior produção de arte como coping durante a pandemia. Além de vídeos e textos, alguns artistas plásticos surgiram no grupo com produções que retratavam as experiências deles. A Figura 3, segundo a autora Deborah Albino, é a reprodução da curva de infecção pelo coronavírus, em que a população espera a queda dela para que o ser encolhido no canto esquerdo possa se liberar da opressão causada pela pandemia. Na subida da curva, há as imagens que marcaram a mortalidade na Itália e chocaram o mundo.

A Figura 4 evidencia, segundo a autora Deborah Albino, a vida por trás das telas de celulares e computadores,

Figura 2. Nuvem de palavras extraídas dos textos produzidos pelo grupo Elam

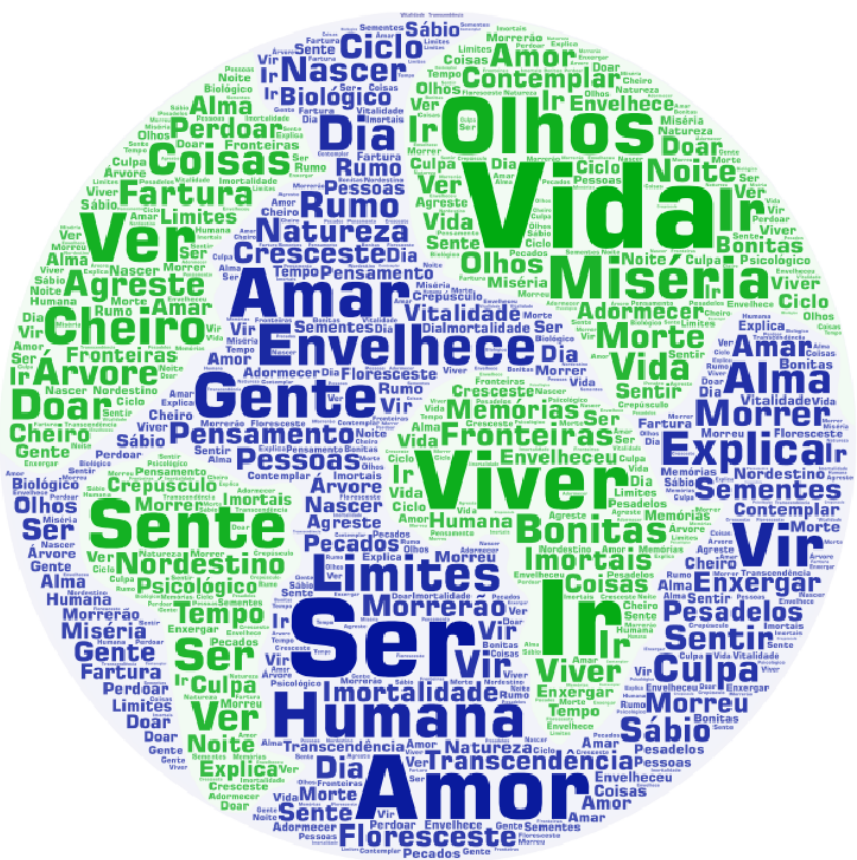

Fonte: Elaborada pelos autores.

Figura 3. Curva da epidemia pela Covid-19 e mortalidade

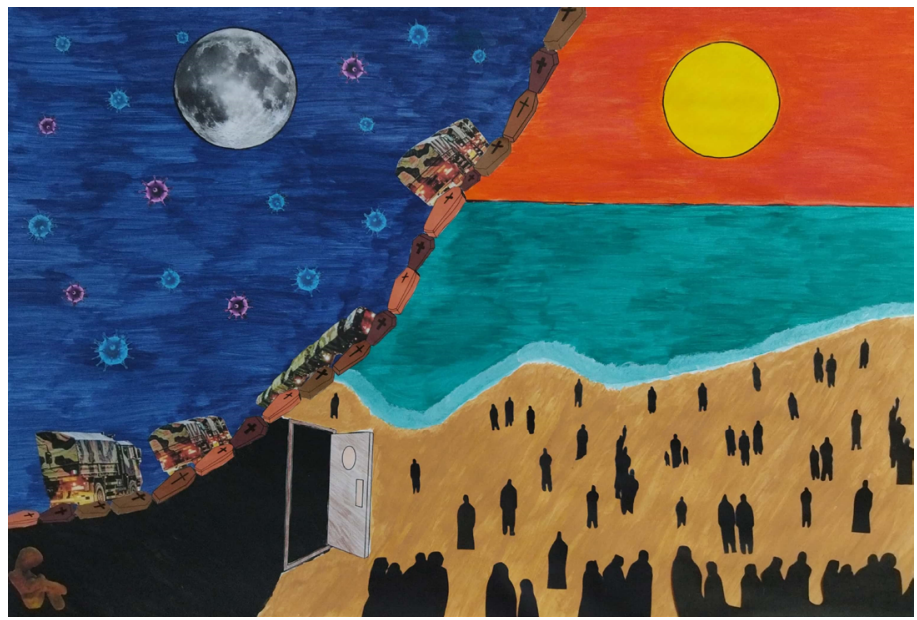

Fonte: Elaborada pela autora da arte Deborah Costa Moreira Albino que passaram a ser o contato com o mundo externo. De um lado, as notícias sobre os casos de Covid-19, as incertezas e a preocupação com a proteção individual, e, do outro, em cores, os familiares e amigos que ajudam as pessoas a atravessar este momento de caos e medo.

A Figura 5 tenta resumir em imagens o que representou a pandemia para os alunos do mundo todo que precisaram reaprender a estudar e interagir. Não apenas um vírus uniu pessoas de diferentes países, mas também a necessidade de encontrar uma forma de seguir em frente, principalmente como estudante de Medicina.

Figura 4. Corredor de telas de computador e celulares com notícias do mundo e das pessoas que permitem o contato com o mundo dentro da quarentena

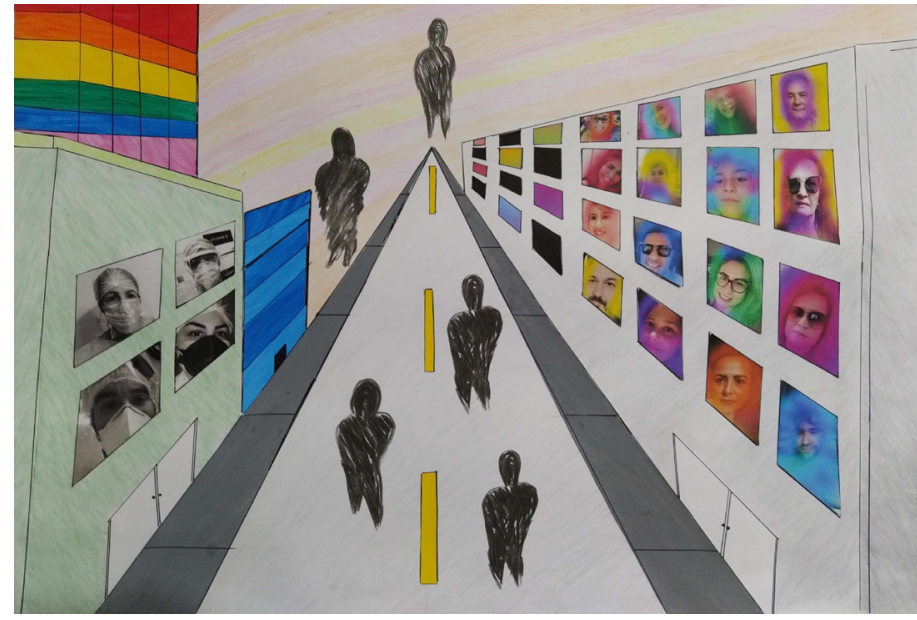

Fonte: Elaborada pela autora da arte Deborah Costa Moreira Albino

Figura 5. Representação da pandemia no mundo e estratégias de conexão

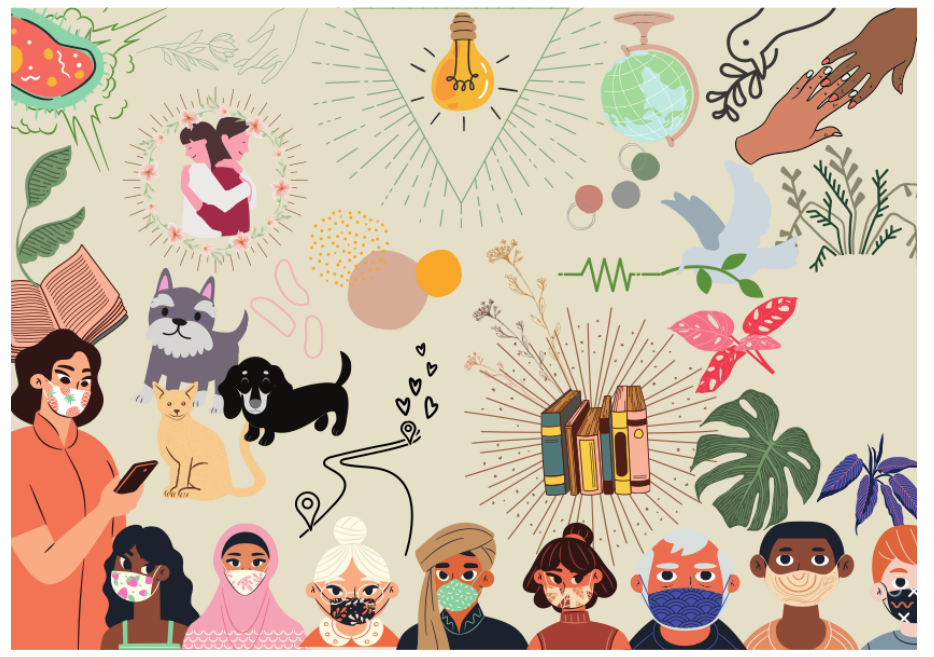

Fonte: Elaborada pela autora da arte Iana Lima Fernandes 


\section{DISCUSSÃO}

O enfrentamento da Covid-19 impõe o isolamento social, o distanciamento físico e a restrição à mobilidade das pessoas como medidas fundamentais para evitar a rápida disseminação do vírus e o crescimento no número de casos da doença. Ressaltamos, no entanto, que essas medidas impactam uma segunda dimensão da vulnerabilidade que merece destaque - a dimensão social -, pois grupos populacionais que já enfrentam historicamente uma trajetória de pressão psicológica sentirão os efeitos da infecção pelo novo coronavírus e do adoecimento pela Covid-19 de forma mais devastadora. Quando o jovem não dispõe de mecanismos internos e/ou externos para lidar com a situação estressante propiciada pela situação, pode haver o desencadeamento de uma série de psicopatologias, como depressão, ansiedade e distúrbios do humor ${ }^{10,11}$.

Eis uma tendência mais ampla na educação médica que se tornou pronunciada na última década: cada vez mais, as escolas de Medicina dos Estados Unidos têm investido em currículo e programação em torno das artes, como a Harvard Medical School e a Escola de Medicina da Dell da Universidade do Texas, em Austin. Os professores argumentam que o envolvimento nas artes durante a faculdade de Medicina é valioso no desenvolvimento de habilidades essenciais de que os médicos precisam, como o pensamento crítico e as habilidades de observação e comunicação, bem como conscientização e empatia. É necessário abordar uma grande variedade de cenários do mundo real, desde a tomada de decisões médicas até a ética. $E$, nessa estrutura, há espaço para o teatro, a música, a literatura e as artes visuais.

A ideia do grupo Elam surgiu pela preocupação dos professores com a ética e o desenvolvimento da consciência e responsabilidade do aluno na graduação e com a futura relação médico-paciente. A perda de empatia tem sido descrita como mecanismo de defesa do estudante mediante seu confronto com realidades de sofrimento e dor dos pacientes a partir do terceiro ano de faculdade. Nossa preocupação também é proteger e manter a empatia dos alunos, de modo que, ao saírem para praticar a medicina, eles ainda sejam indivíduos empáticos ${ }^{12,13}$. Alguns alunos se sentem confiantes com 0 currículo dos primeiros dois anos, mas experimentam maior estresse e ansiedade no início do terceiro ano, o que traz à tona a importância das habilidades interpessoais, habilidade em equipe e flexibilidade durante a rotação por várias especialidades da medicina. O terceiro ano também coloca, inevitavelmente, os estudantes de Medicina em contato com pacientes com doenças terminais. $O$ terceiro ano da faculdade de Medicina foi estudado por Haglund et al.. ${ }^{14}$ No estudo, muitos alunos relataram exposição a traumas, maus-tratos pessoais e péssimos role-models por superiores. A exposição ao trauma foi positivamente associada ao crescimento pessoal no final do ano, o que indica que os alunos tendem a ser resilientes. Em contraste, a exposição a outros eventos estressantes tornou os alunos vulneráveis à depressão e a outros sintomas de estresse ${ }^{14}$. Dyrbye Thomas e Shanafelt ${ }^{15}$ identificaram 40 artigos sobre sofrimento psicológico (ou seja, depressão, ansiedade, esgotamento e problemas de saúde mental relacionados) relacionados a estudantes de Medicina dos Estados Unidos e do Canadá. Os autores descobriram que havia evidências de uma alta prevalência de depressão e ansiedade entre estudantes de Medicina, com níveis de sofrimento psicológico geral consistentemente mais altos do que na população em geral e em pares da mesma idade. No geral, os estudos sugeriram que o sofrimento psicológico pode ser maior entre mulheres estudantes de Medicina ${ }^{15}$.

Para Coleman e Eso-Ahola ${ }^{16}$, o lazer é capaz de gerar mecanismos de coping que ajudam o indivíduo a lidar com os problemas desencadeadores de estresse, assim como o esporte. As concepções de coping, decorrentes do lazer, têm um significado positivo para a saúde, repercutindo na redução dos níveis de depressão, estresse e ansiedade.

$\mathrm{Na}$ cidade de Colúmbia, os alunos podem fazer um curso de quadrinhos ministrado pelo Dr. Benjamin Schwartz, professor assistente de Medicina e diretor criador do Departamento de Cirurgia da Universidade da Colúmbia, que também é cartunista da revista The New Yorker. Em suas aulas de primeiro e quarto anos, os alunos aprendem a criar os próprios quadrinhos e, no processo, obtêm insights sobre as diferentes vantagens de ver e entender as situações da vida real. Talvez o mais importante, eles aprendem a praticar histórias eficazes ${ }^{17}$.

Paracelso já dizia: “Medicina não é apenas uma ciência, é também uma arte”. Ao combinar ciência e arte, a medicina pode ser prolífica na tentativa de investigar e compreender a vida humana como um todo, sem enfocar exclusivamente o estudo de suas partes constituintes.

Autores conduziram um estudo sobre um módulo de artes na Escola Médica de Leicester Warwick e ficaram bastante surpresos ao descobrirem que uma elevada porcentagem dos alunos de Medicina "escrevia poesia, pintava ou tocava música de forma comprometida". Adicionalmente, esses mesmos alunos afirmaram que um dos principais interesses em participar dos grupos de humanidades médicas era para resgatar um antigo interesse que tinha sido deixado de lado, desde que entraram na Escola Médica. Os autores também demonstraram que essa foi uma maneira que os alunos de Medicina encontraram para "manter contato com um 'eu' que a formação médica não mais lhes deixava tempo para, e que sentiam em risco, uma vez que estava distorcido 
pelos constantes 'fatos e números ${ }^{18}$. Foi exatamente o que encontramos no presente estudo, uma surpresa na criação de expressão de arte no grupo inicial do Elam.

Os sintomas de ansiedade e depressão entre estudantes de Medicina são superiores à média encontrada na população em geral, indicando que a faculdade de Medicina pode ser um fator predisponente para essas doenças e suas consequências. Portanto, o cuidado com o aluno tem sido debatido em diversas ambientes, mas o produto final dessa discussão dentro da pandemia atual ainda é incerto ${ }^{19}$.

É importante ressaltar que o sofrimento causado ao aluno tem sido um dos principais fatores para o suicídio no meio médico. Por conta disso, é imprescindível e urgente colocar em prática ações que reduzam a incidência de transtorno mental no curso de Medicina ${ }^{20}$.

\section{CONCLUSÃO}

Em meio à pandemia, sobressai a necessidade de mudança na concepção de qualidade de ensino que tenha como único critério a formação científica do médico e não o bom desenvolvimento e a adaptação biopsicossocial do estudante. Estratégias de enfrentamento de problemas mentais gerados pelo estresse e pela ansiedade no momento de pandemia devem ser construídas para gerar um fator de proteção. É evidente que a saúde mental e o funcionamento psicológico ideal dos estudantes de Medicina são importantes para o treinamento de médicos eficazes. Atravessar esse momento de pandemia pela Covid-19 exigiu uma estratégia de coping para ajudar os alunos a vivenciar e extravasar seus medos, sendo a arte a melhor forma de expressão, além de mais bela, deixando como parte do legado deste momento histórico para as gerações futuras.

\section{CONTRIBUIÇÃO DOS AUTORES}

Melissa Soares Medeiros participou da conceitualização, investigação, escrita do artigo e da metodologia. Dulce Maria Sousa Barreto participou da conceitualização e da visualização. Raquel Sampaio participou da metodologia e da revisão do artigo. Bruno Cavalcante Fales de Brito Alves participou da revisão do artigo e da visualização. Deborah Costa Moreira Albino e lana Lima Fernandes participaram da coleta de dados.

\section{CONFLITO DE INTERESSES}

Os autores declaram não haver conflito de interesses neste estudo.

\section{REFERÊNCIAS}

1. Yaghmour NA, Brigham TP, Richter T, Miller RS, Philibert I, Baldwin DWC Jr., et al. Causes of death of residents in ACGME accredited programs 2000 through 2014: implications for the learning environment. Acad Med. 2017;92(7):976-83. Disponível em: https://pubmed.ncbi.nlm.nih. gov/28514230/.acessado em 20 de Julho 2020

2. Dyrbye LN, Thomas MR, Massie FS, Power DV, Eacker A, Harper W, et al. Burnout and suicidal ideation among U. S. medical students. Ann Intern Med. 2008;149:334-41. doi:10.7326/ 0003-4819-149-5-200809020-00008.

3. Hampton T. Experts address risk of physician suicide. JAMA. 2005;294(10):1189-91. doi: 10.1001/jama.294.10.1189.

4. McCleary-Gaddy AT, Renay S. Addressing mental illness stigma, implicit bias, and stereotypes in medical school. Acad Psychiatry. 2019;43:512-5. doi:10.1007/s40596-019-01081-3.

5. Pacheco JP, Giacomin HT, Tam WW, Ribeiro TB, Arab C, Bezerra IM, et al. Mental health problems among medical students in Brazil: a systematic review and meta-analysis. Rev Bras Psiquiatr. 2017;39:369-78. doi: 10.1590/1516-4446-2017-2223.

6. Tenório LP, Argolo VA, Sá HP, Melo EV, Costa EFO. Saúde mental de estudantes de escolas médicas com diferentes modelos de ensino. Rev Bras Educ Med. 2016;40(4):574-82

7. Cardoso Filho FAB, Magalhães JF, Silva KML, Pereira ISSD. Perfil do estudante de medicina da Universidade do Estado do Rio Grande do Norte(Uern), 2013. Rev Bras Educ Med. 2015;39(1):32-40. doi:10.1590/198152712015v39n1e01092014.

8. Antoniazzi AS, DellAglio DD, Bandeira DR. O conceito de coping: uma revisão teórica. Estud Psicol. (Natal). 1998; 3(2):273-294 acesso em 22 ago 2020. Disponível em: http://www.scielo.br/scielo.php?script=sci arttext\&pid=S1413-294X1998000200006\&lng=en\&nrm=iso\&tlng=pt.

9. Zanini DS, Forns M. Coping y psicopatología: comparación entre adolescentes de la muestra general y sub-clínica. Psiquiatria.com. 2004;8(2). Disponível em: https://psiquiatria.com/trastornos_infantiles/ coping-y-psicopatologia-comparacion-entre-adolescentes-de-lamuestra-general-y-sub-clinica/

10. Zanini DS, Forns $M$, Kirchner T. Coping response and problem appraisal in Spanish adolescents. Percept Mot Skills. 2005;100:153-66. doi: 10.2466/ PMS.100.1.153-166.

11. Peluso MAM, Savalli C, Cúri M, Gorenstein C, Andrade LH. Alterações de humor ao longo da preparação para o vestibular - um estudo longitudinal. Rev Bras Psiquiatr. 2010;32(1):30-6. doi: 10.1590/S151644462010000100007

12. Chen PW. Stories in the service of making a better doctor. The New York Times. 2008 Oct 23 [acesso em 11 jun 2019]. Disponível em: https://www. nytimes.com/2008/10/24/health/chen10-23.html.

13. Tapajós R. Introducing the arts into medical curricula. Interface Comun Saúde Educ. 2002;6(10):27-36. doi: 10.1590/S1414-32832002000100003.

14. Haglund MEM, aan het Rot M, Cooper NS, Nestadt PS, Muller D, Southwick SM, et al. Resilience in the third year of medical school: a prospective study of the associations between stressful events occurring during clinical rotations and student well-being. Acad Med. 2009;84(2):258-68. doi: 10.1097/ACM.obo13e31819381b1.

15. Dyrbye LN, Thomas MR, Shanafelt TD. Systematic review of depression, anxiety, and other indicators of psychological distress among U. S. and Canadian medical students. Acad Med. 2006;81(4):354-73 [acesso em 22 ago 2020]. Disponível em: https://journals.lww.com/academicmedicine/ fulltext/2006/04000/systematic_review_of_depression,_anxiety, and.9.aspx.

16. Coleman D, Eso-Ahola SE. Leisure and health: the role of social support and self-determination. J Leis Res. 1993;25(2):111-28 [acesso em 20 ago 2020]. Disponível em: https://psycnet.apa.org/record/1993-39543-001.

17. Miller E, Balmer D, Hermann N, Graham G, Charon R. Sounding narrative medicine: studying student's professional identity development at Columbia University College of physicians and surgeons. Acad Med. 2014;89(2):335-42. doi: 10.1097/ACM.0000000000000098. 
18. Mairot LTS, Costa BBG, Heringer TPM, Borges RC, Moura EP. Arts in medical education: a systematic literature review. Rev Bras Educ Med. 2019;43(4):54-64. doi:10.1590/1981-52712015v43n4rb20180146.

19. Vasconcelos TC, Dias BRT, Andrade LR, Melo GF, Barbosa L, Souza E. Prevalência de sintomas de ansiedade e depressão em estudantes de medicina. Rev Bras Educ Med. 2015;39 (1):135-42. doi:10.1590/198152712015V39n1e00042014.

20. Martins LAN. Saúde mental do médico e do estudante de medicina. Psychiatry on line Brasil. 1996; 1 (1) [acesso 26 jun 2018]. Disponível em: http://www.polbr.med.br/anog6/saudment.php. 